\title{
A Brief Determination of Certain Class of Power Semicircle Distribution
}

\author{
Rasool Roozegar ${ }^{\dagger, *}$ and Ahmad Reza Soltani ${ }^{\ddagger}$ \\ $\dagger$ Yazd University \\ $\ddagger$ Kuwait University
}

Abstract. In this paper, we give a new and direct proof for the recently proved conjecture raised in Soltani and Roozegar (2012). The conjecture can be proved in a few lines via the integral representation of the Gausshypergeometric function unlike the long proof in Roozegar and Soltani (2013).

Keywords. Power semicircle distribution; Gauss-hypergeometric function; randomly weighted average; arcsine distribution.

MSC 2010: 60E05, 62E15, 33C05.

\section{Introduction}

Following the work of Soltani and Roozegar (2012), consider a randomly weighted average (RWA) of independent and continuous random variables $X_{1}, \ldots, X_{n}$ defined by

$$
S_{n}=R_{1} X_{1}+R_{2} X_{2}+\cdots+R_{n} X_{n}, \quad n \geqslant 2,
$$

where the random proportions $R_{i}=U_{(i)}-U_{(i-1)}, i=1, \ldots, n-1$ and $R_{n}=$ $1-\sum_{i=1}^{n-1} R_{i}$ are the cuts of $(0,1)$ by the order statistics $U_{(1)}, \ldots, U_{(n-1)}$ of a random sample $U_{1}, \ldots, U_{n-1}$ from a uniform distribution on $[0,1], U_{(0)}=0$ and $U_{(n)}=1$. Following Roozegar and Soltani (2013) specify certain classes of power semicircle laws that are randomly weighted average distributions. More precisely, they show that the power semicircle (PS) distribution with

* Corresponding author 
shape parameter $\theta\left(\theta>-\frac{3}{2}\right)$ and range parameter $\sigma(\sigma>0)$, whose density is given by

$$
C_{\theta, \sigma}\left(\sigma^{2}-x^{2}\right)^{\theta+\frac{1}{2}}
$$

where $C_{\theta, \sigma}=\frac{1}{\sqrt{\pi} \sigma^{2 \theta+2}} \cdot \frac{\Gamma(\theta+2)}{\Gamma\left(\theta+\frac{3}{2}\right)}$ and $x \in(-\sigma, \sigma)$ is indeed a RWA distribution for $\theta=\frac{n-3}{2}$ and $n \geqslant 2$. This result is proved by Soltani and Roozegar (2012) just for $n=2,3,4$. The long proof of this result which is given in Roozegar and Soltani (2013) for all $n$ is based on the formula for the Stieltjes transform (ST) of a PS distribution. This formula is derived by Arizmendi and Perez-Abreul (2010), namely,

$$
\mathcal{S}\left[F_{\theta}\right](z)=(\theta+1) \int_{0}^{1}(1-t)^{\theta}\left(z^{2}-t \sigma^{2}\right)^{\frac{-1}{2}} d t, \quad \theta>-1 .
$$

Soltani and Roozegar (2012) indicate,

$$
\mathcal{S}\left[F_{S_{n}} ; n\right](z)=\left[\mathcal{S}\left[F_{X_{1}}\right](z)\right]^{n}, \quad z \in \mathbb{C} \bigcap_{i=1}^{n}\left(\operatorname{supp} F_{X_{i}}\right)^{c},
$$

where $\mathcal{S}[H](z)$ and $\mathcal{S}[H ; \rho](z)$ are ST and generalized ST of a distribution $H$, respectively. These transforms are defined by

$$
\mathcal{S}[H](z)=\int_{\mathbb{R}} \frac{1}{z-x} H(d x), \quad z \in \mathbb{C} \cap(\operatorname{supp} H)^{c},
$$

and

$$
\mathcal{S}[H ; \rho](z)=\int_{\mathbb{R}} \frac{1}{(z-x)^{\rho}} H(d x), \quad z \in \mathbb{C} \cap(\operatorname{supp} H)^{c}, \rho>0,
$$

where $\mathbb{C}$ is the set of complex numbers, supp $H$ stands for the support of $H$ and $\rho$ is a constant.

The main objective here is to employ the Gauss-hypergeometric function ${ }_{2} F_{1}$, which is defined by the series

$$
{ }_{2} F_{1}(c, a ; b ; z)=\sum_{n=0}^{\infty} \frac{(c)_{n}(a)_{n}}{(b)_{n} n !} z^{n}
$$

where $(a)_{0}=1$ and $(a)_{n}=a(a+1)(a+2) \ldots(a+n-1), n \geqslant 1$, denotes the rising factorial. Gauss-hypergeometric function ${ }_{2} F_{1}$ has the Euler's integral representation of the form

$$
{ }_{2} F_{1}(c, a ; b ; z)=\frac{\Gamma(b)}{\Gamma(a) \Gamma(b-a)} \int_{0}^{1} \frac{t^{a-1}(1-t)^{b-a-1}}{(1-z t)^{c}} d t .
$$


For more details on Gauss-hypergeometric function and its properties, see Abramowitz and Stegun (2012) and also Andrews et al. (1999).

\section{PS Distribution}

In this section we present a short proof of the following theorem of Roozegar and Soltani (2013).

Theorem 1. Let the random variables $X_{1}, \ldots, X_{n}$ be independent and have common arcsine distribution on $(-\sigma, \sigma), \sigma>0$. Then $S_{n}$ will have a PS distribution on $(-\sigma, \sigma)$ with $\theta=\frac{n-3}{2}, n \geqslant 2$.

Proof. Without loss of generality, we assume $\sigma=1$. For the arcsine distribution $(\theta=-1$ in PS distribution), we have

$$
\mathcal{S}\left[F_{-1}\right](z)=\frac{1}{\sqrt{z^{2}-1}}
$$

In view of (3), it suffices to show that

$$
a_{n} \int_{-1}^{1} \frac{1}{(z-x)^{n}}\left(1-x^{2}\right)^{\frac{n-2}{2}} d x=\left(\frac{1}{\sqrt{z^{2}-1}}\right)^{n}
$$

where $a_{n}=\frac{\Gamma\left(\frac{n+1}{2}\right)}{\sqrt{\pi} \Gamma\left(\frac{n}{2}\right)}$.

Indeed, for any $n \geqslant 2$, one has for $|z|>1$, via change of variables $t=\frac{x+1}{2}$,

$$
\begin{aligned}
a_{n} \int_{-1}^{1} \frac{1}{(z-x)^{n}}\left(1-x^{2}\right)^{\frac{n-2}{2}} d x & =b_{n} \int_{0}^{1} \frac{1}{(z+1-2 t)^{n}}\{t(1-t)\}^{\frac{n-2}{2}} d t \\
& =\frac{b_{n}}{(z+1)^{n}} \int_{0}^{1} \frac{1}{\left(1-\frac{2 t}{z+1}\right)^{n}}\{t(1-t)\}^{\frac{n-2}{2}} d t
\end{aligned}
$$


where $b_{n}=\frac{2^{n-1} \Gamma\left(\frac{n+1}{2}\right)}{\sqrt{\pi} \Gamma\left(\frac{n}{2}\right)}$. Now applying (4), we arrive at

$$
\begin{aligned}
\int_{-1}^{1} \frac{1}{(z-x)^{n}}\left(1-x^{2}\right)^{\frac{n-2}{2}} d x & \propto \frac{1}{(z+1)^{n}}{ }_{2} F_{1}\left(n, \frac{n}{2} ; n ; \frac{2}{z+1}\right) \\
& =\frac{1}{(z+1)^{n}} \sum_{n=0}^{\infty} \frac{\left(\frac{n}{2}\right)_{n}}{n !}\left(\frac{2}{z+1}\right)^{n} \\
& =\frac{1}{(z+1)^{n}} \cdot \frac{1}{\left(1-\frac{2}{z+1}\right)^{\frac{n}{2}}} \\
& =\left(\frac{1}{\sqrt{z^{2}-1}}\right)^{n} .
\end{aligned}
$$

This completes the proof.

\section{Acknowledgement}

The authors are very grateful to the Referees and Editor in Chief for valuable suggestions and comments which greatly improve the paper. The first author is also thankful to the Yazd University for supporting this research.

\section{References}

Abramowitz, M. and Stegun, I.A. (2012). Handbook of Mathematical Functions: with Formulas, Graphs, and Mathematical Tables. Courier Dover Publications.

Andrews, G.E., Askey, R. and Roy, R. (1999). Special Functions. Cambridge University press.

Arizmendi, O. and Perez-Abreu, V. (2010). On the Non-Classical Nnfinie Divisibility of Power Semicircle Distributions. Comm. Stoch. Annal., 4, 161-178.

Roozegar, R. and Soltani, A.R. (2013). Classes of Power Semicircle Laws That Are Randomly Weighted Average Distributions. J. Stat. Comput. Simul., to appear. doi: 10.1080/00949655.2013.806510.

Soltani, A.R. and Roozegar, R. (2012). On Distribution of Randomly Ordered Uniform Incremental Weighted Averages: Divided Difference Approach. Statist. Probab. Lett., 82, 10121020. 


\section{Rasool Roozegar}

Department of Statistics, Yazd University,

Yazd, Iran.

email: rroozegar@yazd.ac.ir

\author{
Ahmad Reza Soltani \\ Department of Statistics and Operations Research, \\ Kuwait University, \\ Safat, Kuwait. \\ email: soltani@kuc01.kuniv.edu.kw
}

